\title{
The Making of 3D Microscopic Movies: A Look Behind the Scenes of the Fast Tomographic Imaging Program at TOMCAT
}

\author{
$\underline{\text { Christian M. Schlepütz }}{ }^{1,}{ }^{*}$ Anne Bonnin ${ }^{1}$, Federica Marone $^{1}$ and Marco Stampanoni ${ }^{1,2}$
}

1. Swiss Light Source, Paul Scherrer Institute, 5232 Villigen PSI, Switzerland

2. Institute for Biomedical Engineering, ETH Zurich, 8092 Zurich, Switzerland

${ }^{*}$ Corresponding author, christian.schlepuetz@psi.ch

When going to the cinema and watching a blockbuster movie, it is often hard to fathom the amount of work, effort and meticulous planning required to make a story come to life in a motion picture until the movie is over and the end credits are scrolling over the screen in a seemingly endless progression. Being able to engage top actors is definitely a great asset, but by no means a sufficient condition to guarantee success.

While the fast tomography program at the TOmographic Microscopy and Coherent rAdiology experimentTs (TOMCAT) beamline [1] of the Swiss Light Source (SLS, Paul Scherrer Institut, Villigen, Switzerland) is certainly not producing big screen motion pictures, it is equally true that, although being the very essence of any scientific project, a great idea by itself is not yet a sufficient condition for a successful experiment. We heavily rely on a multitude of different technical developments, a reliable and robust infrastructure, and a dedicated team of specialists from various disciplines to make our experiments work and to keep pushing the boundaries of what can be achieved with time-resolved high-resolution imaging.

In this talk, I will present an overview of the different building blocks, both in terms of the available equipment as well as the infrastructure required to seamlessly integrate these individual components into a working experimental setup, that were adopted or developed at TOMCAT and PSI to advance our capabilities in acquiring high-speed tomographic data sets, thus allowing us to observe microscopic dynamic phenomena in 3D and real-time. Recent progress in pushing the time-resolution beyond its current limits as well as a collection of as of yet unpublished new experimental results on different dynamic systems from research fields as diverse as biomechanics, pharmaceutical sciences, metal processing, and food science will be presented to illustrate these advances.

The fast tomography program at TOMCAT has been consistently built up over nearly a decade with dedicated efforts to develop and implement the hardware and equipment necessary to run such a program. A major milestone and breakthrough for high-speed imaging has been the in-house development of the GigaFRoST camera and readout system [2], enabling streaming data acquisition at very high sustained data rates of up to $8 \mathrm{~Gb} / \mathrm{s}$. Combined with the recent procurement of a high numerical aperture microscope (4x magnification), this allows for a merely continuous observation of dynamic phenomena over their full duration with superb image quality even at very low exposure times. These advances in the detection system have been accompanied by crucial upgrades and developments of the sample holder and environments, providing support for applications as diverse as in vivo imaging of small rodents under lifesupporting conditions [3] or infrared laser-based sample heating up to $1700^{\circ} \mathrm{C}$ [4].

One very important ingredient for dynamical experiments, particularly if they are run in a retrospective gating mode, where the obtained projections are to be sorted according to their time point within a quasi- 


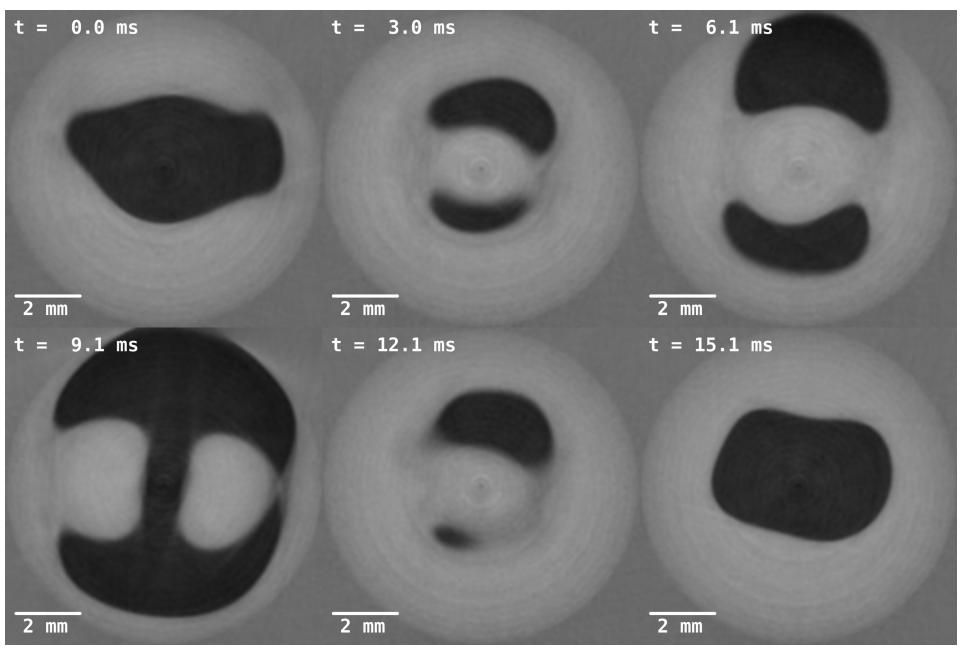

Figure 1: Tomographic axial slices through the air-water interface of a $41.25 \mathrm{~Hz}$ standing wave inside a round water vial at different time points during the periodic motion cycle. Air is shown by dark gray levels, while the water appears in light gray. periodic or periodic motion cycle, is the ability to record various stimulation or response signals synchronously with the high-speed imaging process. Thanks to the availability of a host of signal processing, synchronization, and recording tools [3], this task has become common practice during fast experiments and has proven to be extremely valuable for the data analysis. For example, Figure 1 shows the water-air interface of a standing water wave inside a round water vial, which was mounted on a loudspeaker membrane. The standing wave was produced by an external sinusoidal drive signal, whose phase with respect to the individual image frames acquired at $2 \mathrm{kHz}$ frame rate was precisely determined from the recorded drive and response signals.

One often overlooked aspect of taking large amounts of high-speed data is the fact that the way a user can and needs to interact with the data during an experiment is very different from traditional static tomography. Keeping up with the incoming data stream to produce reconstructed data sets is practically impossible as scan times (measured in fractions of a second) become significantly shorter than the time required to reconstruct (typically several tens of seconds). Over and above that, a direct reconstruction may not be possible in many cases as additional and non-standard data processing steps (e.g.: retrospective gating, resorting, partitioning, motion correction, etc.) or the application of more advanced reconstruction algorithms (e.g.: iterative methods) are required. In fact, many projects rely on custom analysis procedures which can only be fully developed once the data has been acquired. This has two immediate consequences: (i) The direct feedback obtainable from the data during the execution of an experiment or even a whole campaign is at best partial. Even for cases where the reconstruction is straight-forward in principle, typically only a subset of the data can be made available in reconstructed form within a reasonable time frame after a measurement. Simple visual inspection of the volume data may also not be sufficient to detect the dynamics of the sought-out structural changes, raising the need for automated evaluation routines. Taking advantage of the availability of a real-time data stream, we are currently developing methods to run simple and configurable metrics on partial data streams coming directly from the camera, to perform full reconstruction directly on the streamed data [5], as well as a nearly real-time reconstruction of a few representative slices through the volume. (ii) Since the reconstruction and processing takes a lot of time and may actually only happen after the proper methods have been developed on a small subset of the acquired data, large amounts of raw data need to be kept on storage and be made accessible to the users even after the end of the experimental campaign, i.e., from off-site. PSI is currently implementing the necessary infrastructure to manage and permanently archive these data and to provide the required remote access capabilities to process and analyze data on PSI compute clusters [6], thus removing the need to transfer all data to a user's home institution. A simplified sketch of the infrastructure and data flows is shown in Figure 2 [7]. 


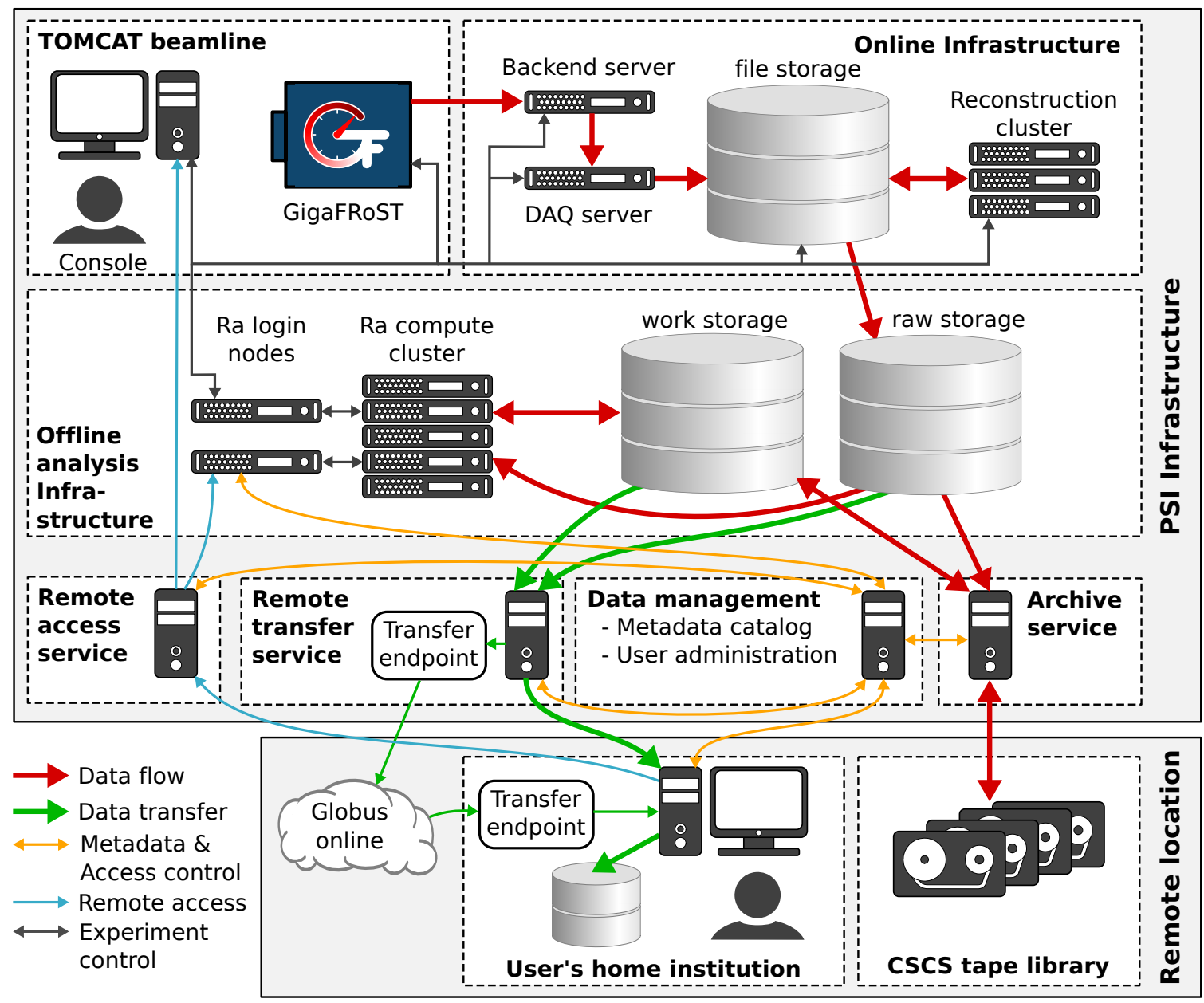

Figure 2: Sketch of the IT infrastructure put in place at PSI for users to facilitate access to large data volumes and provide high-performance compute-resources on-site for users which do not have access to such infrastructure at their home institutions.

\section{References:}

[1] M. Stampanoni et al, Proceeding of SPIE (2006) p. 63180M.

[2] R. Mokso et al, J. Synchrotron Rad. 24 (2017) 1250.

[3] G. Lovrić et al, Physica Medica 32 (2016) 1771.

[4] J. L. Fife et al, J. Synchrotron Rad. 19 (2012) 352.

[5] F. Marone et al, Adv. Struct. Chem. Imag. 3 (2017) 1.

[6] https://www.psi.ch/photon-science-data-services/photon-science-data-services

[7] The authors acknowledge the TOMCAT beamline technicians for superb support in building up the equipment for fast tomography experiments, as well as the IT and controls specialists who ensure a tight integration of new gadgets into the beamline controls system and provide innovative software developments to unlock ever new features of our devices. 
https://doi.org/10.1017/S1431927618014484 Published online by Cambridge University Press 